\title{
Bringing Worth Maps a step further: A dedicated Online-Resource
}

\author{
Fatoumata G. Camara ${ }^{\mathrm{a}}$, Gaëlle Calvary ${ }^{\mathrm{b}}$ \\ Univ. Grenoble Alpes, CNRS, Grenoble INP ${ }^{1}$, LIG, F-38000 Grenoble, France \\ afatoumatag.camaralgmail.com, bgaelle.calvary@imag.fr
}

\begin{abstract}
Worth Maps (WMs) are promising because they model interactive systems following different perspectives. Consequently, WMs support design in many ways. ARROW was introduced to provide designers with a systematic approach to worth mapping. However, the framework currently remains untested, which raises open questions about general applicability and relevance.

In this work, we operationalize ARROW in additional design cases. With insights gained from the operational experience, we propose ARROWS (for ARROW-Support) as a refinement of the initial framework. ARROWS was assessed via a workshop with designers. Results highlight the need for appropriate resources supporting worth mapping. In order to fulfill this need, we have created and released a website providing designers with knowledge on ARROWS and WMs that follows a more practically oriented perspective.
\end{abstract}

Keywords: Worth-Centered Design (WCD), Worth Maps (WMs), ARROW, ARROWS, online resource

\section{Introduction}

Since it is now clear that design must go beyond usability, we have witnessed the introduction of different approaches to designing for human-oriented attributes. For instance, the framework for positive experience [25] makes the assumption that the fulfillment of ten basic human needs leads to positive user experience (UX). These ten basic needs are: 'Security', 'Keeping the meaningful', 'Relatedness', 'Popularity', 'Competition', 'Physical health', 'Competence', 'Influence', and 'Stimulation'. The framework for positive experience takes other aspects into account, such as usability and accessibility. Value-Sensitive Design (VSD) proposes including all values in design and especially those with moral import [15, 16]. In [16], Friedman and colleagues highlight a list of twelve values which are often implicated in interactive systems design. These twelve values are: 'Human welfare', 'Ownership and property', 'Privacy', 'Freedom from bias', 'Universal usability', 'Trust', 'Informed consent', 'Accountability', 'Courtesy', 'Identity', 'Calmness', and 'Environmental sustainability'. As witnessed by the aforementioned list, usability does matter in VSD. It is,

\footnotetext{
${ }^{1}$ Institute of Engineering Univ. Grenoble Alpes
} 
however, differentiated from values and refers to criteria that characterize the system from a functional point of view.

Other work, with similar goals (i.e., designing for human-centered attributes), consider a larger scope of aspects. For instance, Almquist and colleagues [1] identified thirty elements of value coming into play when customers evaluate a product or service. These thirty elements are organized in a pyramidal hierarchy around four categories, which are (from bottom to top): 'Functional' (14 elements) (e.g., 'Save time', 'Sensory appeal'), 'Emotional' (10) (e.g., 'Nostalgia', 'Design/aesthetics'), 'Life changing' (5) (e.g., 'Heirloom', 'Self-actualization'), and 'Social impact' (e.g., 'Selftranscendence'). Worth-Centered Design focuses on worth $[6,7,8]$, defined as 'things that will motivate people to buy, learn, use or recommend an interactive product, and ideally most or all of these' [8]. Worth can be modeled using Worth Maps as a connection between system-oriented and human-oriented elements. As such, from 'designing as crafting' WMs shift design to 'designing as connecting': they represent an interesting design tool.

This work is related to worth maps. The design tool has been investigated in several projects $[2,9,11,13,14,19,27]$. If there is a consensus on worth maps benefits for design, examples of existing WMs are more diverse in terms of constituent elements. In order to shape the content of WMs, Camara and Calvary [2] proposed the ARROW framework and illustrated it using a concrete case. However, beyond, the ARROW framework has not received yet much attention.

More precisely, this work addresses the general applicability and relevance of ARROW. Firstly, we have used the ARROW framework to create WMs in different project contexts. Secondarily, we have conducted a workshop with designers to assess insights gathered from the first worth mapping experience. Contributions are the following.

- We prove the applicability and relevance of ARROW.

- We present 'ARROW-Support (ARROWS)' to propose refinements and extensions for the ARROW framework.

- We prove the understandability of ARROWS as well as, once more, the relevance of WMs for design.

- We propose an online resource dedicated to ARROWS and WMs.

The remainder is organized as follows: The next section discusses relevant literature associated with this work. Section 3 relates our worth mapping experience with a focus on Lyric, the illustrative case. Section 4 presents propositions of ARROWS for refining and extending the ARROW framework. Section 5 describes the assessment of ARROWS through a workshop conducted with designers. Section 6 presents the online resource dedicated to ARROWS and WMs. Finally, section 7 concludes the paper and highlights directions for future work. 


\section{$2 \quad$ Literature Review}

\subsection{Worth Maps}

WMs are inspired from Hierarchical Value Maps (HVMs) [21] used in marketing to study customers' motivations for purchase. HVMs combined separately elicited Means-Ends Chains (MECs) together. MECs, in turn, connect (product) attributes (A) to (usage) consequences (C) and consequences to (personal) values (V): MECs are then formed of A-C-V chains [17].

WMs connect system-oriented attributes (also referred as 'design elements') to human-oriented ones (also referred as 'human elements'). Examples of design elements include materials and features; examples of human elements are usage impacts, feelings, and needs. WMs support three of the WCD meta-principles [12] ('expressivity', 'credibility', and 'committedness') and consider both positive and negative aspects related to the interactive system. Positive connections explain why users would buy, learn, use, and/or recommend the interactive system; negative ones explain factors that might hinder the use of the interactive system.

WMs have a vertical representation; the positive generally appears upwards while the negative appears downwards.

In interactive systems design, WMs were first called 'Worth/Aversion Maps (W/AMs)'. W/AMs moved HVMs from release to early design stages (such as 'opportunity identification' [7]) [10]. W/AMs also revisited HVMs in several points [10, 13].

- In HVMs, consequences can be functional or psychosocial; W/AMs refined and extended these with further types.

- In HVMs, values are restricted to the 18 instrumental and 18 terminal values from Rokeach's Value Survey [22]; W/AMs imposed no vocabulary in regards to values formulation.

- In HVMs, functional consequences must precede psychosocial ones; W/AMs do not impose such a rigid ordering.

In [13], W/AMs are revised and re-baptized 'worth maps'. Changes mainly occurred regarding two points. Firstly, W/AMs had retained the two types of product attributes in HVMs: concrete and abstract attributes. WMs refined concrete attributes into two types: materials and features. Moreover, positive and negative abstract attributes are in WMs re-named respectively to qualities and defects. Secondarily, an alternative structure, handling user experience as interplay of feelings and actions, are used for usage consequences in WMs.

In [2], the authors relate their complete operationalization of the WCD framework through the development of Cocoon, phase by phase. In order to fulfill the lack of a concrete methodology for worth mapping, faced at 'design' phase, the authors propose the ARROW (Appreciations, Requirements and Rationale Of Worth) frame- 
work, which also suggests different types of classes of elements and connections for WMs. The ARROW framework is at the core of this work and is described in more detail in the next section.

\subsection{The ARROW Framework}

ARROW (initially PEW [3]) was developed during the development of Cocoon, a mobile and context-aware application. The ARROW framework addresses worth as a twofold notion: 'Appreciated Worth' vs. 'Requested Worth' [2].

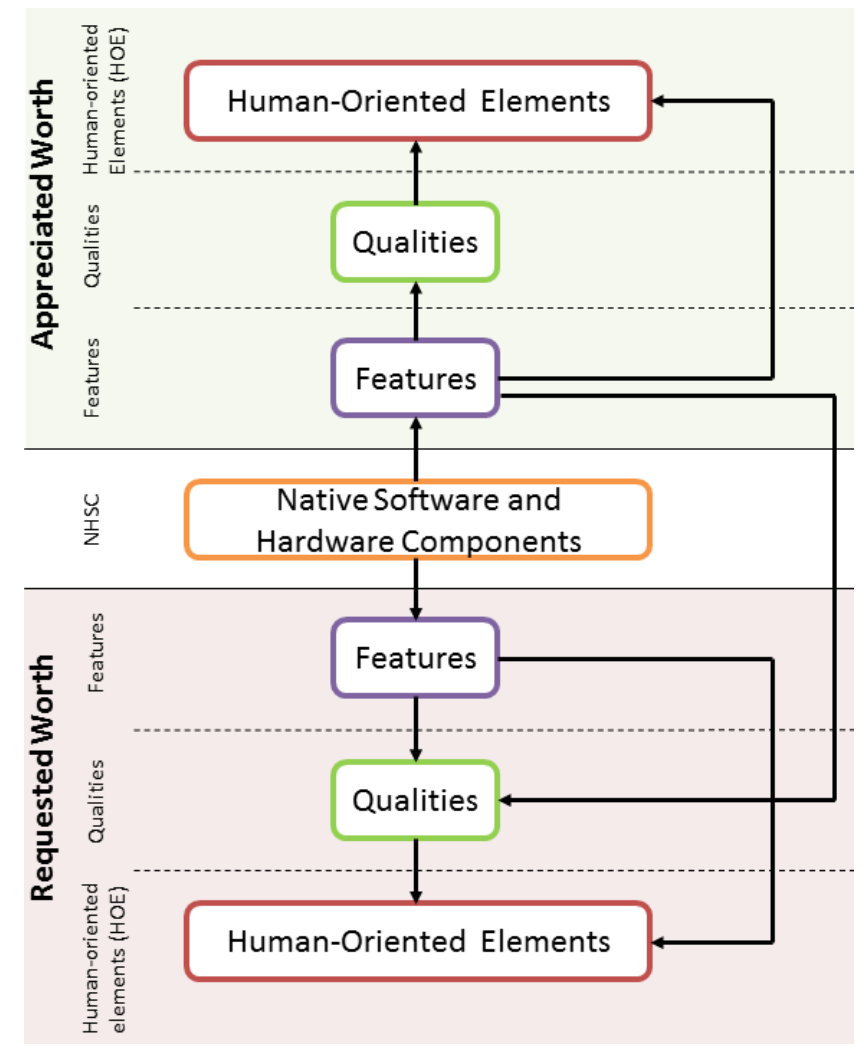

Fig. 1: The ARROW Framework

Appreciated Worth represents the positive aspects of the interactive system (i.e., the strengths). By referring to worth definition, Appreciated worth highlights factors that motivate the user to buy, learn, use and/or recommend the interactive system.

Requested Worth overcomes the negative aspects of the interactive system (i.e., the weaknesses). In other words, through Requested worth, ARROW suggests that WMs rather highlight compensatory elements instead of negative aspects themselves. 
For instance, let's imagine that users point out information redundancy as a discouraging factor for the system use. Instead of representing 'information redundancy' in the worth map, ARROW suggests considering a means for controlling information frequency.

ARROW proposes that WMs are structured around Appreciated worth, Requested worth, and a third additional class of elements: 'Native Software and Hardware Components (NSHC)' of the device(s) hosting interaction. The framework proposes further that both Appreciated worth and Requested worth consider three sub-categories of elements: 'Features' (of the interactive system), 'Qualities' (of Features), and 'Human-Oriented Elements (HOE)' (impacted by interaction).

ARROW also suggests a vertical representation for WMs: Appreciated worth on the upper part, Requested worth on the lower part, and NSHC in the middle as they may support both appreciated and requested features.

\subsection{WMs in Design}

Previous work shows that WMs support design in several ways. In [13], authors conducted a field study with 11 families in order to investigate whether WMs could help highlight design solutions for family archiving purposes. In [14], sentence completion supported worth mapping with the aim to understand deeper motivations of online players. In both works, worth mapping has led to profitable outcomes for projects globally, worth mapping as an approach, and to WMs in terms of content.

Otero et al. conducted five semi-structured interviews to support the worth mapping process during the development of a digital public display for a teachers' common room $[19,20]$. In conclusions, the authors state: 'The exercise of explicitly stating the connections between features, qualities, and higher-level constructs about use fostered critical thinking and search for alternative design solutions'. Vu relied on a user study consisting of interviews to populate two WMs which were used for the main purpose of WMs: connect design sensitivities to human ones [27].

In [2] also, worth mapping is combined with other HCI techniques for data collection purposes: semi-structured interviews at the 'study of needs' phase and a field study followed by group interviews at 'evaluation' phase. Camara and Calvary showed that WMs could support: User Interface (UI) and interaction design, graphic design, and software implementation. In addition, because WMs based on ARROW are symmetric, the authors relied on an approach based on WMs comparison to support the evaluation of Cocoon. Finally, they highlight WMs as support to communication in heterogeneous design teams, thanks to their visual representation (i.e. expressivity of WMs).

WMs have evolved considerably over the time. Derived from HVMs, they were initially introduced as W/AMs, W/AMs were in turn refined and, more recently, ARROW was introduced as a framework dedicated to shape the content of WMs. If ARROW seems to be well suited for WMs in interactive systems, the framework 
presentation lacks detailed information regarding classes of elements and connections between them. Therefore, further goals of our work are to find out more about the ARROW classes and to understand the types of possible relationships that may link elements to each other.

\section{Worth mapping beyond Cocoon}

\subsection{The design cases}

The design cases considered for the worth mapping experience were selected in order to investigate worth maps in different application domains and design stages (see Table 1): Lyric $^{2,3}$ (a heating controller system), Colibri ${ }^{4}$ (an advanced planning system) [4], FutureID ${ }^{5}$ (an identity management system), and Cocoon (a mobile and context-sensitive system) [2]. Furthermore, our literature review revealed that most worth mapping experiences relied on user-centered approaches to collect worth elements and understand connections between them (see section 2.3). Yet, users' involvement requires resources (e.g., in terms of time and expertise) which may not be available in every project. Therefore, during this exercise, we have made the choice not to use any user-centered approach with the aim to test out whether worth mapping could be successful without support from users.

Here, we focus on our illustrative case, Lyric. Below, we describe the system as well as the worth mapping process and its outcomes. More information regarding the remaining cases is available in the online resource (see section 6).

\begin{tabular}{|l|c|c|c|c|}
\hline \multicolumn{1}{|c|}{ Domain } & $\begin{array}{c}\text { Lyric } \\
\text { Design stage }\end{array}$ & $\begin{array}{c}\text { Colibri } \\
\text { (Suppy Chain) }\end{array}$ & $\begin{array}{c}\text { FutureID } \\
\text { (Identity) }\end{array}$ & $\begin{array}{c}\text { Cocoon } \\
\text { (Adaptation) }\end{array}$ \\
\hline Opportunity identification & & - & & \\
\hline Analysis & & & & - \\
\hline Design & & & & - \\
\hline Evaluation & & & & \\
\hline Release & $\mathbf{n}$ & & & \\
\hline
\end{tabular}

Table 1: Design cases characterization according to application domain and design stage.

\subsection{Lyric: The illustrative case}

Lyric is a heating system controller that offers a great deal beyond the primary functions of a thermostat through a distributed interaction between the Lyric device

\footnotetext{
${ }^{2}$ https: / / www youtube. com/watch? $\mathrm{v}=\mathrm{HU} 0 \mathrm{y} 5 \mathrm{vFdUz} 8$

${ }^{3}$ https : / / www . youtube. com/watch? $=$ =yKKrFaJbRy 8 \& feature=youtu. be

${ }^{4}$ https : / / www . youtube. com/watch? $=I$ zc $8 H j e K 3 w Q \&$ feature=youtu. be

5 http://www. futureid.eu/
} 
and the user's mobile devices (Smartphone and tablet). With Lyric, the user has the possibility to set the temperature to a desired value; the system takes different parameters (home temperature, humidity and outdoor weather) into account in order to perform the request. The user has also the possibility to set the thermostat to 'away'; the system knows then when the home is occupied vs. empty and uses the user's location to proceed to appropriate adjustments according to user's preferred settings ('Geofencing'). Additionally, Lyric allows the user to check the weather and create 'Shortcuts', which are easy to use custom settings (for instance, temperature at $60^{\circ}$ on Fridays from 8:30 AM when the home is empty).

Lyric was chosen as an illustrative example because the heating controller system was used to motivate the first work around worth. Indeed, in [5], Cockton recalls reasons that might motivate for controlling heat in the home (e.g., money saving, care for the environment). Further, the author explains that existing systems (at that time) do not deliver the true worth by stating 'I know of no central heating controller that tells me how much money (and fuel) I've saved by setting a particular program'. Therefore, more than ten years later, it would be interesting to see whether current systems address missing features to meet users' intrinsic motivations.

We have collected information from the Honeywell website and different videos to construct a worth map for Lyric. This information has allowed us to receive insights regarding Lyric features (as mentioned above) as well as regarding different aspects for populating the other layers of the worth map (NSCHs, qualities, and HOEs). It is, however, important to note that these sources mainly focus on positive aspects, corresponding only to the upper side of the worth map (i.e., Appreciated Worth). Nevertheless, it was straightforward to notice that Lyric does not let the user know the amount of money and/or energy s/he saved thanks to the effort put into programming the heating system. As a consequence, the 'Money/Energy savings computation' feature could be evidently considered as part of Requested Worth for Lyric. Additional research, supported by existing literature related to worth/values, has allowed us to identify additional Requested Worth elements.

The worth map for Lyric (see Fig. 2) diagrammatically summarizes the strengths of the thermostat. As such, it could support communication: proper advisement towards customers and efficient communication within the design team. Through identified missing aspects, the worth map also provides the design team with directions for improving the product during an iteration phase. 


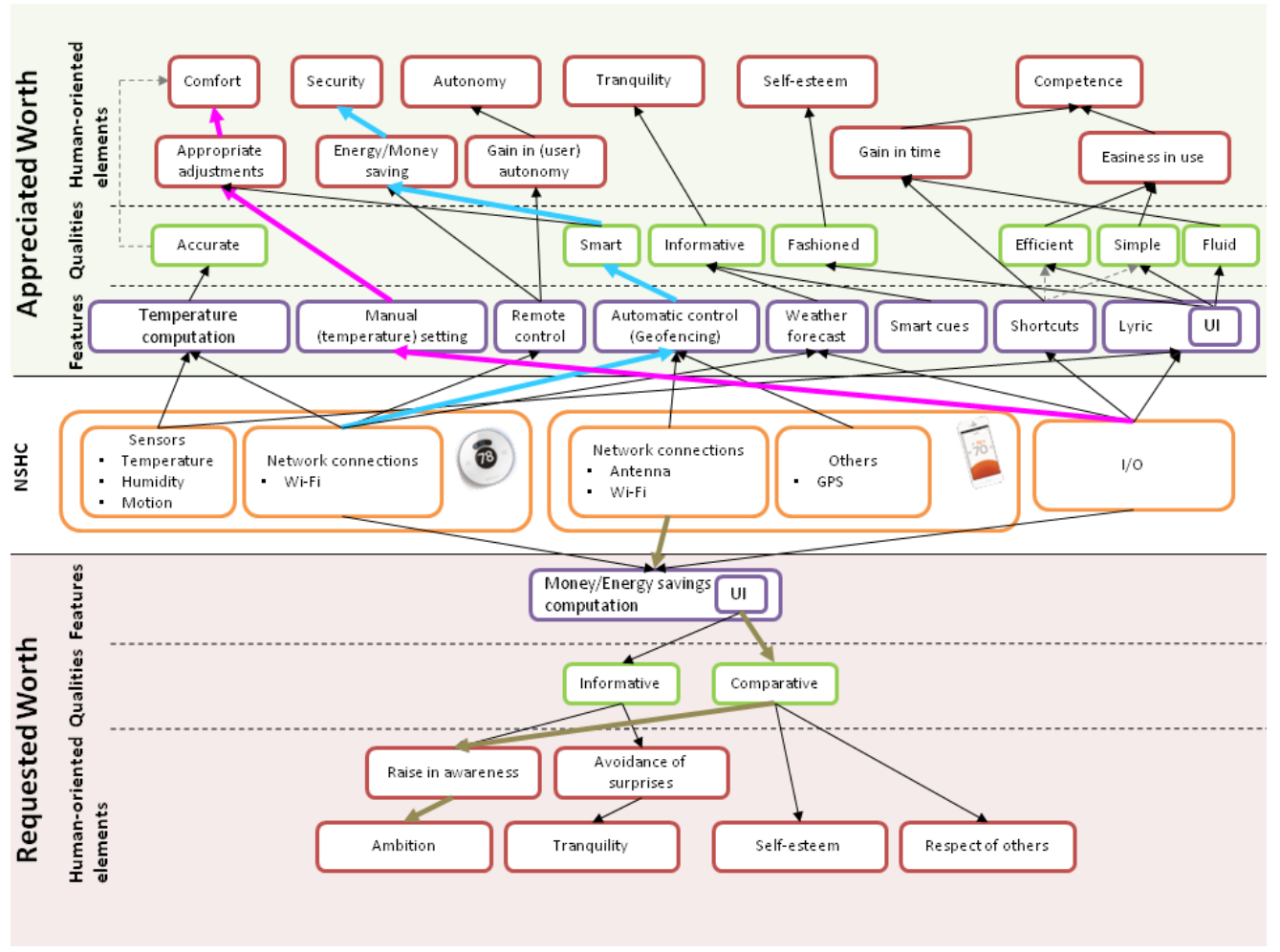

Fig. 2: The Lyric worth map

The NSHC layer illustrates modularization: native software and hardware components are grouped according to types of device.

The highlighted chains can be read as suggested below.

- Blue: the Geofencing feature relies on some network connections (the Wi-Fi of the Lyric device is highlighted here) for data exchange; the feature presents the quality of being smart as it knows when the house is empty or occupied and proceeds to appropriate adjustments accordingly; as such, the Geofencing feature contributes to energy and money saving, which may enhance financial security for the user.

- Pink: the manual setting feature allows the user to perform appropriate adjustments (thanks to input devices) and, therefore, allows her to experience physical comfort through adapted room temperatures.

- Gray: the Money/Energy saving computation component would rely on wireless network connections for data exchange; the feature should present the quality of being comparative, i.e., provide the user with comparable information (e.g., energy consumptions over the last three months, total of energy consumption over the last six months in comparison to the carbon footprint of a flight Paris- 
Berlin); such information might raise the users awareness of energy consumption and, consequently, create ambition for a change of habit.

\subsection{Outcomes}

The entire worth mapping experience was carried out over three years and four application domains. Six WMs were constructed in total: 4 from scratch (1 for Lyric; 2 for Colibri; 1 for FutureID) and 2 as revisions of first versions for Cocoon. Thanks to this outcome, we can conclude that worth mapping can be successful without users' involvement.

Our worth mapping experience also shows that ARROW, as originally described, can be applied to diverse domains. However, while progressing in the journey with WMs, we have gained more understanding on the framework and WMs in general and could, therefore, identify opportunities for improvement. In the next section, we detail propositions for refining/extending the ARROW framework.

\section{ARROW-Support}

ARROW-Support (ARROWS) aims at supporting the ARROW framework by providing definitions and additional knowledge regarding classes of elements and connections in WMs. Furthermore, ARROWS explicitly introduces 'Modularization' $[13,14]$ as a feature of WMs.

\subsection{Classes of Elements}

ARROW defines three first-level categories: Appreciated Worth, Requested Worth, and Native Software Hardware Components (NSHC); both Appreciated and Requested worth are structured around three sub-classes: Features, Qualities, and Human-Oriented Elements (HOEs). ARROWS keeps the same classes of elements.

- Native Software Hardware Components (NSHC): we define NSHC as peripheral components that can support the interactive system both from an internal (i.e., underlying processes) and an external point of view (i.e., the UI). Standard input and output (I/O) devices, such as the mouse, the keyboard, the camera, and the microphone, are generally part of NSHC. In the specific case of Lyric, we can, in addition, mention the GPS of the mobile device (considering that the system could rely on it to locate the user) as well as the Wi-Fi for dataexchange.

- Features: we propose considering the interactive system in detail by examining each feature individually, but, also globally by examining it as a whole. In the specific case of Lyric, examples of individual features include: the automatic temperature control by the system (Geofencing) as well as the manual tempera- 
ture setting by the user. The global feature representing the system as a whole is named as the system, so 'Lyric' in the illustrative example. Furthermore, ARROWS suggests paying a particular attention to the UI (and the associated interaction) by considering it as a sub-feature of each feature.

- Qualities: we propose that WMs distinguish four types of qualities:

* UI and Interaction qualities highlight dimensions of usability, ergonomic criteria as well as aesthetic aspects related to the interactive system. In Lyric, such qualities include: 'Simple', 'Efficient' or 'Fashioned'.

* Inherent qualities result from the spirit of a feature or a concept. Inherent qualities can also be related to a company identity or culture and could, therefore, highlight dimensions of brand experience (BX) [23]. In the specific case of Lyric, 'Smart' represents such a quality since the thermostat has initially been designed to be so.

* Functional qualities are related to underlying processes sustaining the UI and the interaction (e.g., data computation, database access). In the case of Lyric, 'Accurate' belongs to functional qualities since the system takes different parameters into account in order to compute the temperature as accurately as possible. 'Instantaneous' could also be part of Lyric (appreciated) functional qualities if changes requested remotely (from the mobile phone, for instance) are taken into account within a relative short period of time (e.g., $0,5 \mathrm{~s})$.

* Global qualities reveal characteristics related to the interactive system considered as a whole (i.e., related to the global feature). Because only stability of many features makes a whole system stable, 'Stable' can be considered a global quality in many cases, including the specific one of Lyric.

It is important to note that a global quality can be UI-related, inherent, or functional. In the case of Lyric, 'Fashioned' is a UI-related global quality and 'stable' could be considered as inherent-global one if the development team had the aim to design a system with an exceptional degree of stability and had, therefore, made effort to reach this goal, for instance, through specific testing strategies. In another example, 'Expensive' could be considered as an inherent quality of Apple products since high cost seems to be part of the company's culture that contributes to the brand image (for ensuring high quality). However, since expensiveness is related to the product as a whole, 'Expensive' would be a inherent-global quality.

It might be difficult sometimes to label a quality: there can be a fuzzy line between the aforementioned classes of quality. Therefore, we recommend practitioners rely on the three questions in order to determine more easily whether a quality is inherent, UI/Interaction-related, functional and/or global. 


\begin{tabular}{|l|l|l|}
\hline \multicolumn{3}{|c|}{ Inherent qualities } \\
\hline $\begin{array}{l}\text { Is the quality very specific to the context (e.g., system, project, } \\
\text { company)? }\end{array}$ & Yes & \\
\hline \multicolumn{2}{|c|}{ Some UI/Interaction-related and functional qualities } \\
\hline Is the quality objectively measurable? & Yes & \\
\hline \multicolumn{2}{|c|}{ Global qualities } & No \\
\hline Is the quality related to a specific feature? & \\
\hline
\end{tabular}

Table 2: Helper for determining qualities types

Table 2 highlights that inherent qualities are not generally objectively measureable, UI-related qualities can be objectively measurable and functional ones are generally objectively measurable. Additionally, we recommend practitioners proceed to qualities labeling according to: from inherent to UI-related/functional and from specific to global.

- Human-Oriented Elements (HOEs): as in the initial framework, ARROWS represents human-oriented attributes under the name 'Human-Oriented Elements (HOEs)'. User experience, understood as the result of direct interaction with a product or a service and indirect interaction (through a third party), is part of HOEs. It is important to note that interaction also includes here both anticipated and post usage. HOEs also encompass interaction consequences as well as higher-level elements impacted by interaction (though consequences or not), such as human needs and values.

In the specific case of Lyric such elements include (financial) 'Security' thanks to energy saving, (physical) 'Comfort' thanks to appropriate temperature adjustments, and (feeling of) 'Competence' since the user can easily reach his/her goals thanks to the simple and efficient UI.

\subsection{Connections between elements}

Let's consider that features, qualities, and human-oriented elements part of Appreciated worth are respectively represented by: AF ('Appreciated Feature'), AQ, and AHOE. The same naming rules apply to Requested worth. The types of connection depicted on the ARROW framework (see Fig. 1) can be textually translated as follows.

$$
\begin{aligned}
& -(1) \mathrm{NHSC} \rightarrow \mathrm{AF} \rightarrow \mathrm{AQ} \rightarrow \mathrm{AHOE} \\
& -(2) \mathrm{NHSC} \rightarrow \mathrm{RF} \rightarrow \mathrm{RQ} \rightarrow \mathrm{RHOE} \\
& -(3) \mathrm{NHSC} \rightarrow \mathrm{AF} \rightarrow \mathrm{AQ} \\
& -(4) \mathrm{NHSC} \rightarrow \mathrm{RF} \rightarrow \mathrm{RQ} \\
& - \text { (5) NHSC } \rightarrow \mathrm{AF} \rightarrow \mathrm{AHOE} \\
& - \text { (6) NHSC } \rightarrow \mathrm{RF} \rightarrow \mathrm{RHOE}
\end{aligned}
$$


ARROW suggests full but also partial chains within WMs. If full chains make complete sense ((1), (2)), partial ones are more debatable since they assume that features with good qualities can contribute to worth even if they don't impact any HOE (i.e., (3), (4)) and that human-oriented elements can be directly impacted through features without intermediary qualities (i.e., (5), (6)).

Indeed, the worth mapping experience allowed us to understand that every feature presents one or more qualities and that every quality impacts human elements. As a consequence, WMs would only be composed of complete chains. Nevertheless, we could also admit that partial chains can be relevant in some cases: when the humanattribute element is reached though an expected quality (the connection between the quality and the HOE can be omitted) and when the human-attribute element is reached though an intrinsic (and not differentiating) quality (the connection between the feature and the quality can be omitted).

For instance, in the Lyric worth map (see Fig. 2), the quality 'Accurate' (of the feature 'Temperature computation') could be linked to the human-oriented element ('Comfort') considering that the user would be able to make good provisions accordingly. However, since accuracy would be expected from every thermostat, the impact in terms of HOE can be assumed as not significant and, as a consequence, the corresponding connection omitted.

\subsection{Modularization}

During the worth mapping exercise, we have understood that WMs can and should be modularized, both at elements and connections levels. Indeed, in [13], the authors grouped worth elements due a lack of space and, in [14], researchers use Microsoft Office Visio drawing layers to group complex connections. The worth mapping experience revealed that other reasons could, however motivate, modularity in WMs.

Worth maps can be rather big, which negatively impacts readability and understandability. Partial chains can already contribute to reduce complexity. Additionally, thanks to modularity, WMs could first give an overview before progressively disclosing details, as suggested by the information visualization mantra: 'overview first, zoom and filter, then details-on-demand' [24]. By doing so, WMs could also support the WCD meta-principles of 'inclusiveness' in an appropriate way by adapting information-level in the different layers according to stakeholders.

\section{$5 \quad$ Workshop with Designers}

To assess the meaningfulness and relevance of knowledge produced during the worth mapping experience, we conducted a one-day workshop, which provided us with the opportunity to introduce ARROWS and WMs to a group of practitioners. 


\subsection{Participants}

Six people (4 female, 2 male) from a telecommunication company and all involved in interactive systems design participated in the workshop. The group of practitioners included: two human factors experts, a research project manager, an innovative service designer, a communication service architect, and a psychologist. According to data collected, participants had diverse backgrounds and played different roles in design.

\subsection{Procedure}

During the workshop, the participants were divided into two teams: the project manager, the innovative service designer, and one human factors specialist together in a first team; the communication service architect, the psychologist, and the other human factors specialist in a second team. The following activities were carried out (in the order they appear).

- Self-Introduction: participants as well as facilitators first built their personal and professional profile using Lego bricks. Then, each person commented on her tangible representation to introduce his/herself.

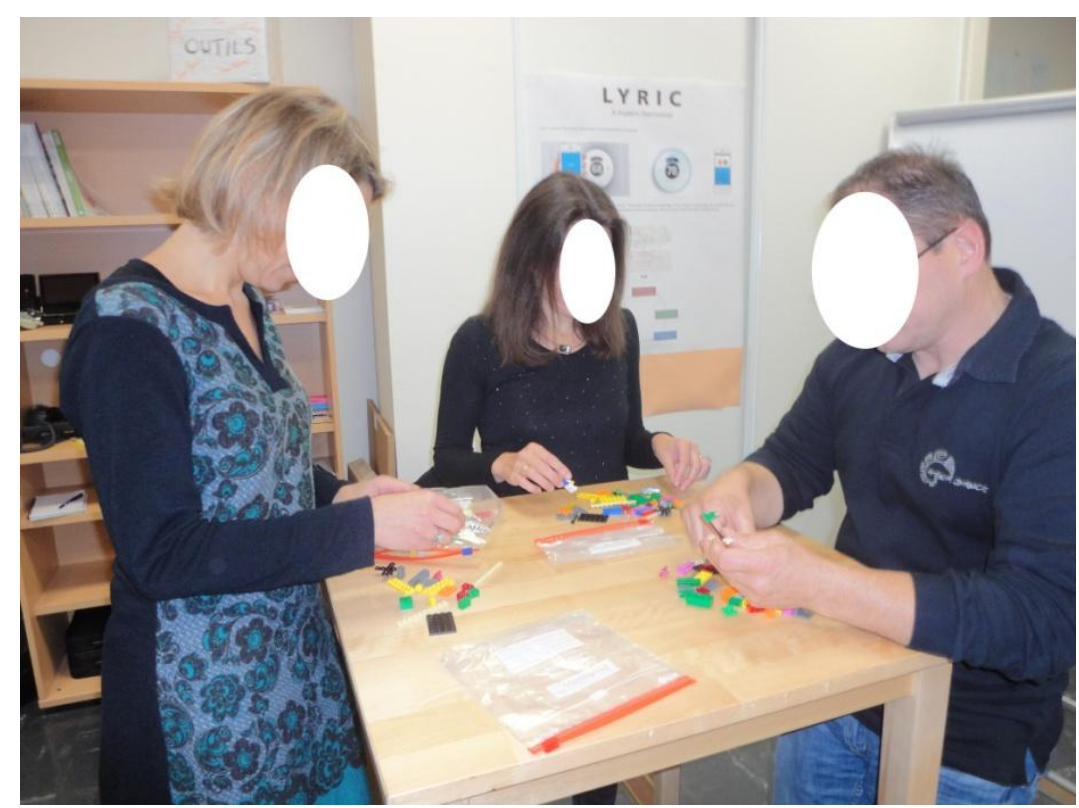

Fig. 3: A group of participants building their profile with Lego bricks

- Refection on 'Worth': each participant materialized 'worth' using Lego bricks and, then, commented to share his/her perspectives on the notion with others. 
- Design of the ideal thermostat: first, each team built an ideal thermostat using Lego bricks. Second, a team member described the resulting system from each side. Third, the two teams provided each other with feedback.

- Presentation of Lyric: the Lyric thermostat was introduced to participants through two videos.

- Introduction to ARROWS and WMs: WMs were first briefly defined. Then, ARROWS was introduced using Lyric. During the presentation, participants were allowed to interrupt the speaker to ask questions or to comment.

- Interactive Poster Session: WMs resulting from the worth mapping experience were presented during a poster session where participants and workshop facilitators could exchange. A poster representing each design case was presented. For each case, the poster described the project context and explained when (i.e., design stage(s)) and how they were used (i.e., purpose(s)).

The entire session was video-recorded. After the workshop, all the material used, including the posters, was sent to participants as attachment to a thank-you message. Participants were also invited to fill a questionnaire online, which was structured around three points.

1. Participants' data: to request information related to participants' gender, background, experience and roles in interactive systems design.

2. Worth maps: to assess the understandability of ARROWS and WMs as well as to collect participants' opinions regarding the relevance of worth maps for design and factors that could hinder their use in actual projects.

3. Lego bricks: to understand the gap that may exist between a representation built upon Lego bricks and information that can be captured from it.

This paper focuses only on the first two points. Related outcomes based both on information collected through questionnaires and insights gathered during the workshop are presented in the next section.

\subsection{Outcomes}

\section{Participants' profile}

The collected data shows that workshop participants have diverse backgrounds. Thanks to their previous experiences, participants appeared to be equipped with different skills: strong technical ones but with a good understanding of Human Factors (HF) or the opposite. Finally, participants have declared taking part in different design stages, in which they play different roles.

\section{Understandability of ARROWS and WMs}

In order to verify that participants have fully understood information conveyed during the workshop, we have analyzed their definition of WMs. Only one participant (the architect) did not provide a (proper) definition for WMs. Instead, he formulated the following statement: 'It's complicated to define, however do we really need a well formalized definition?'. 
The other participants defined WMs as follows.

'A means for connecting users' primary needs to sensors and other technical components through intermediary features and qualities that foster paying attention to both existing and missing aspects (features, qualities, and values) and supports assessment of the interactive system's state at a given time' (human factors expert 1).

'A cartography evolving over time and that represents the different values of a product/service and for monitoring possible improvements' (project manager).

'A good means of giving design a more profound dimension, thanks to the investigation beyond features and qualities' (innovative service designer).

'A more complete way to model and represent an interactive system; a means for addressing user experience more globally (not only focused on the UI)' (human factors expert 2).

'A cartography that allows to visualize relationships between the user's values and a product/service' (psychologist).

Participants' definitions highlight that they have different perspectives on WMs, which is not surprising considering their different jobs and backgrounds. Nevertheless, the statements show that participants gained a good understanding of WMs as well as of the underlying ARROWS, providing that definitions surface: the structure using different layers, the focus on both design and human elements and the aim to connect them, and attention to aspects beyond the UI.

It is important to note that analysis of other answers from the architect show that he also understood well ARROWs and WMs. For instance, he wrote the following as a motivator for worth maps use: '... because they highlight connections between the designed object and more profound values, both conscious and unconscious for the user'.

\section{Relevance of WMs for design}

Participants' motivations for WMs use highlight that they perceive the relevance of WMs for design. Indeed, participants would investigate worth maps because they would allow them to: visualize connections between design and human elements; identify strengths, weaknesses, and missing aspects; give attention to elements that are sometimes forgotten (such as emotions); prioritize features; test out something new (i.e., novelty).

In the questionnaire, participants were requested to identify design activities which can be well supported by WMs and explain how they would use them. Table 3 summarizes participants' responses. 


\begin{tabular}{|l|l|l|l|l|l|l|}
\hline \multicolumn{1}{|c|}{ Design stage } & $\begin{array}{l}\text { Opportunity } \\
\text { Identification }\end{array}$ & $\begin{array}{l}\text { Study of } \\
\text { Needs }\end{array}$ & $\begin{array}{l}\text { Software } \\
\text { Architecture }\end{array}$ & $\begin{array}{l}\text { UI } \\
\text { Design }\end{array}$ & $\begin{array}{l}\text { Graphic } \\
\text { Design }\end{array}$ & $\begin{array}{l}\text { Evalua- } \\
\text { tion }\end{array}$ \\
\hline HF Expert 1 & Yes & & Yes & Yes & Yes & Yes \\
\hline Manager & & Yes & & Yes & & Yes \\
\hline Service designer & Yes & Yes & & & & \\
\hline Architect & Yes & Yes & & & & Yes \\
\hline HF Expert 2 & Yes & Yes & & & & Yes \\
\hline Psychologist & Yes & Yes & & & Yes & Yes \\
\hline
\end{tabular}

Table 3: Design activities that can be well supported by WMs according to participants

According to participants, WMs use is most relevant at 'Opportunity Identification', 'Study of Needs', and 'Evaluation' phases. Participants also think that WMs could substantially support the creation of UI, as well as of graphic design. Software development-related activities are, according to participants, the ones that could be supported by WMs use in the less relevant way. Indeed, only one participant (i.e., one of the human factor specialists) pointed out that WMs could be used for the software architecture elaboration. It is important to note that this participant has a technical background. Furthermore, if the work in [3] highlights WMs as a means to support software implementation, according to our data, none of the participants would rely on WMs during software implementation. However, the project manager insisted on the fact that WMs could be well suited for agile developments as a monitoring tool from one sprint to another.

Our findings confirm that WMs can, indeed, support design in different ways. From our understanding, WMs can be seen as 'Boundary Objects' [26] for multidisciplinary design teams, from which everyone can retrieve a minimum of information relevant to his/her concerns, and which contribute to enhancing understanding (and, therefore, designing worthwhile systems) and facilitating communication (and, therefore, reinforcing unity in heterogeneous settings).

\section{Factors hindering WMs use}

Participants' answers regarding factors that could hinder WMs use in actual design projects are the following.

'Maybe the time required to set them up' (human expert 1).

'Difficulties and costs related to initial worth map creation; lack of explanations available for the entire project team; required additional costs and competencies' (project manager).

'We need to have time as well as necessary competencies for it' (innovative service designer).

'The time required to properly assimilate the concept and to get enough experience in order to take advantage of the method' (service architect).

'It doesn't seem easy to start from scratch for the design of a new system' (human factors expert 2). 


\section{‘Cumbersome operationalization' (psychologist).}

Verbatims above show that the effort, in terms of time and intellectual demands, required for worth mapping might hinder use of WMs in actual design projects. Participants' statements also highlight uncertainties regarding the ability for an independent use of WMs (as already identified in [13]).

We believe that appropriate resources could help overcome these issues and, as a first step, we have designed and released a website dedicated to WMs. Next section presents this online resource for WMs.

\section{The Online Resource}

Our online resource is accessible at: http: / /phdgirl911.wixsite.com/arrows-and-wms. It provides designers with more practice-oriented knowledge on ARROWS and worth maps: the user walks through ARROWS step-by-step thanks to the Lyric example, which is introduced on a dedicated page. A particular section explains the benefits of WMs for design using concrete cases. In addition, the user is provided with the possibility to download the ARROWS template, examples of WMs, and the posters used during the workshop with designers.

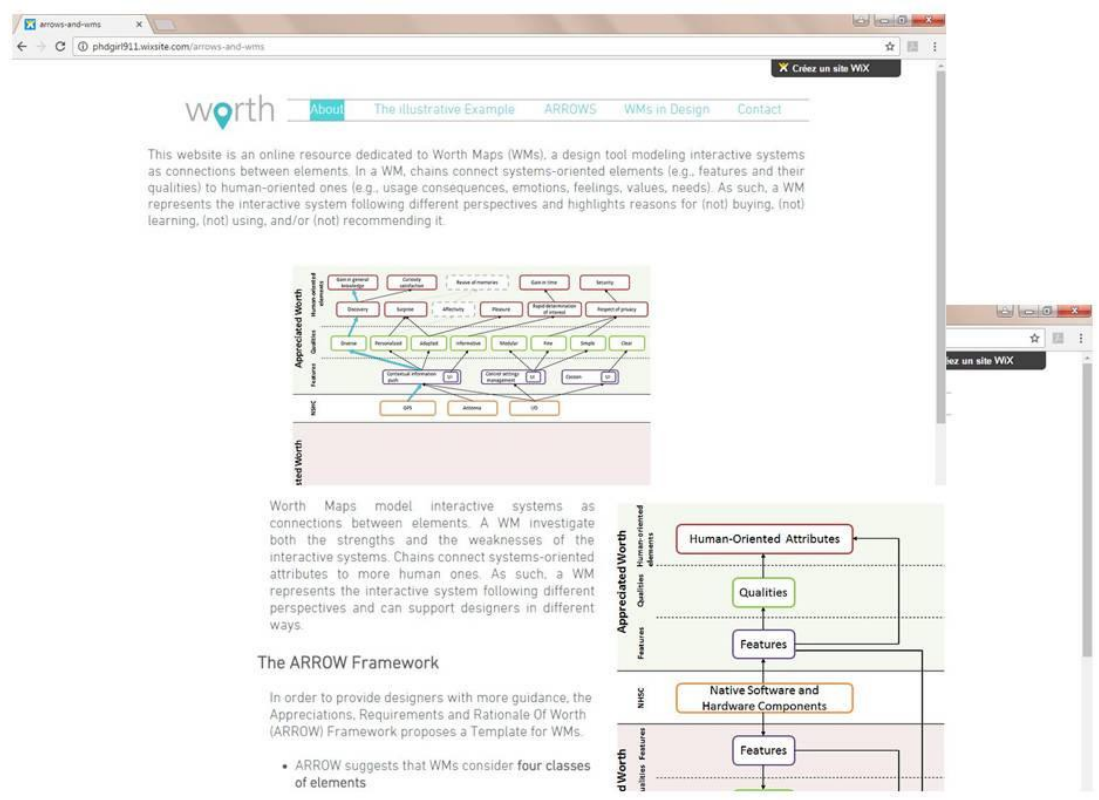

Fig. 4: The website dedicated to ARROWS and WMs 


\section{$7 \quad$ General Discussion and Conclusion}

Based on the observation that the ARROW framework has not yet received much attention, we have engaged in the creation of WMs in different design projects using ARROW. This worth mapping experience provided us with more insights, which allowed us to refine/extend the ARROW framework and propose ARROW-Support (ARROWS). ARROWS was assessed through a workshop with designers. Concerns of participants of the workshop highlighted the necessity to make appropriate resources available in order to encourage the use of WMs. Therefore, we have designed and released a website to support designers getting started with WMs.

ARROWS defines the classes of elements that should be considered in WMs, details connections to be investigated between elements, and provides support for labeling qualities. Our experience shows that ARROWS is well appropriated for structuring worth maps in interactive systems design. Nevertheless, during the worth mapping experience, it was sometimes debatable whether a specific element belonged to a specific class or to another: the classification task was not always straightforward. Therefore, it is important to note that worth mapping should be flexible since the worth mapping process as well as worth maps (in terms of layout and content) can be sensitive to the design context. For instance, as highlighted in this paper, researchers' backgrounds (and maybe sensitivities also) can have an impact on worth elements naming and classification (see sections 1, 4.1). According to Cockton and colleagues, the worth mapping process needs to be adapted to participants [13]. In our opinion, worth mapping and WMs could and should be adapted to a broader range of aspects (e.g., application domain, goals, people, and design phase).

Even though several factors can influence the worth mapping process and WMs, we believe that it exists a classification of worth elements (for instance, according to device types or application domains). However, only a more extensive use of worth maps could help elaborate such a classification (i.e., ontology for worth). In addition to allowing us to get a better understanding of worth, a worth ontology would support new areas of research, such as persuasive technologies [18], which rely on worth but suffer from the lack of visibility and maturity of methods and techniques for worthcentered developments.

Hopefully, our online resource will contribute to a broader use of WMs. However, due to the lack of an appropriate tool, the construction of WMs will probably still remain problematic. Therefore, in the future, we will focus on the development of a tool for an interactive creation of WMs. This tool will also offer features for copying, duplicating, and comparing WMs.

This paper presents a wide range of ways to use WMs in design. However, all of them suggest a use of WMs at design time. To the best of our knowledge, WMs have not been, so far, investigated at runtime. Therefore, in the future, we will also focus on WMs as support to adaptativity. 


\section{Acknowledgment}

We warmly thank Orange Labs and Fraunhofer IAO for their participation and support.

\section{References}

1. Almquist, E., Senior, J., Bloch, N.: The elements of value. Harvard Business Review (2016), https://hbr.org/2016/09/the-elements-of-value

2. Camara, F., Calvary, G.: Worth-centered design in practice: Lessons from experience and research agenda. In: Human-Computer Interaction - INTERACT 2015

3. Camara, F., Calvary, G., Demumieux, R.: The pew framework for worth mapping. In: Kotz, P., Marsden, G., Lindgaard, G., Wesson, J., Winckler, M. (eds.) Human-Computer Interaction - INTERACT 2013

4. Camara, F., Parmentier, T., Kharab, M.: Colibri: Towards a new generation of advanced planning systems. 25me conférence francophone sur l'Interaction Homme-Machine, IHM'13 (2013)

5. Cockton, G.: From quality in use to value in the world. In: CHI'04 Extended Abstracts on Human Factors in Computing Systems. pp. 1287-1290. CHI EA'04, ACM, New York, NY, USA (2004)

6. Cockton, G.: Value-centred hci. In: Proceedings of the Third Nordic Conference on Human-computer Interaction. pp. 149-160. NordiCHI '04, ACM, New York, NY, USA (2004)

7. Cockton, G.: A development framework for value-centered design. In: CHI'05 Extended Abstracts on Human Factors in Computing Systems. pp. 1292-1295. CHI EA'05, ACM, New York, NY, USA (2005)

8. Cockton, G.: Designing worth is worth designing. In: Proceedings of the 4th Nordic Conference on Human-computer Interaction: Changing Roles. pp. 165-174. NordiCHI'06, ACM, New York, NY, USA (2006)

9. Cockton, G.: Designing worth-connecting preferred means to desired ends. interactions 15(4), 54-57 (Jul 2008)

10. Cockton, G.: Putting value into e-valu-ation. In: Law, E.C., Hvannberg, E., Cockton, G. (eds.) Maturing Usability, pp. 287-317. Human-Computer Interaction Series, Springer London (2008)

11. Cockton, G.: Sketch worth, catch dreams, be fruity. In: CHI'08 Extended Abstracts on Human Factors in Computing Systems. pp. 2579-2582. CHI EA'08, ACM, New York, NY, USA (2008)

12. Cockton, G.: Getting there: Six meta-principles and interaction design. In: Proceedings of the SIGCHI Conference on Human Factors in Computing Systems. pp. 2223-2232. CHI'09, ACM, New York, NY, USA (2009)

13. Cockton, G., Kirk, D., Sellen, A., Banks, R.: Evolving and augmenting worth mapping for family archives. In: Proceedings of the 23rd British HCI Group Annual Conference on People and Computers: Celebrating People and Technology. pp. 329-338. BCS-HCI'09, British Computer Society, Swinton, UK, UK (2009)

14. Cockton, G., Kujala, S., Nurkka, P., Hltt, T.: Supporting worth mapping with sentence completion. In: Gross, T., Gulliksen, J., Kotz, P., Oestreicher, L., Palanque, P., Prates, R., Winckler, M. (eds.) INTERACT 2009. Lecture Notes in Computer Science, vol. 5727, pp. 566-581. Springer Berlin Heidelberg (2009) 
15. Friedman, B.: Value-sensitive design. interactions 3(6), 16-23 (1996)

16. Friedman, B., Kahn Jr, P.H., Borning, A., Huldtgren, A.: Value sensitive design and information systems. In: Early engagement and new technologies: Opening up the laboratory, pp. 55-95. Springer (2013)

17. Gutman, J.: A means-end chain model based on consumer categorization processes. The Journal of Marketing pp. 60-72 (1982)

18. Laurillau, Y., Foulonneau, A., Calvary, G., Villain, E.: Sepia, a support for engineering persuasive interactive applications: Properties and functions. In: Proceedings of the 8th ACM SIGCHI Symposium on Engineering Interactive Computing Systems (EICS'16). pp. 217-228. ACM (2016)

19. Otero, N., José, R.: Worth and human values at the centre of designing situated digital public displays. Int. J. Adv. Pervasive Ubiquitous Comput. 1(4), 1-13 (2009)

20. Otero, N., Rego, A., José, R.: Considering the inclusion of worth and values in the design of interactive artifacts. In: Proceedings of the First International Conference on Integration of Design, Engineering and Management for Innovation (IDEMI2009) (2009)

21. Reynolds, T.J., Gutman, J.: Laddering theory, method, analysis, and interpretation. Journal of advertising research 28(1), 11-31 (1988)

22. Rokeach, M.: The nature of human values, vol. 438. Free press New York (1973)

23. Roto, V., Lu, Y., Nieminen, H., Tutal, E.: Designing for user and brand experience via company-wide experience goals. In: Proceedings of the 33rd Annual ACM Conference Extended Abstracts on Human Factors in Computing Systems. pp. 2277-2282. CHI EA'15, ACM, New York, NY, USA (2015)

24. Shneiderman, B.: The eyes have it: A task by data type taxonomy for information visualizations. In: Proceedings of the 1996 IEEE Symposium on Visual Languages. VL '96, IEEE Computer Society, Washington, DC, USA (1996)

25. Sonnleitner, A., Pawlowski, M., Ksser, T., Peissner, M.: Experimentally manipulating positive user experience based on the ful_lment of user needs. In: Kotz, P., Marsden, G., Lindgaard, G., Wesson, J., Winckler, M. (eds.) Human-Computer Interaction INTERACT 2013, Lecture Notes in Computer Science, vol. 8120, pp. 555-562. Springer Berlin Heidelberg (2013)

26. Star, S.L., Griesemer, J.R.: Institutional ecology, 'translations' and boundary objects: Amateurs and professionals in berkeley's museum of vertebrate zoology. Social studies of science 19(3), pp. 387-420 (1989)

27. Vu, P.: A Worth-Centered Development Approach to Information Management System Design. Master's thesis, Aalto University (2013) 